\title{
Femoral neck fractures in osteogenesis imperfecta patients: A retrospective case series and novel surgical technique
}

\author{
Mark T. Gorsche, Michelle S. Caird, Frances A. Farley, G. Ying Li, Matthew D. Abbott* \\ Department of Orthopaedic Surgery, Michigan Medicine, Ann Arbor, MI, Unites States
}

Received: May 4, 2017

DOI: $10.5430 /$ css.v3n3p14
Accepted: July 13, 2017

URL: https://doi.org/10.5430/css.v3n3p14
Online Published: August 7, 2017

\begin{abstract}
Case: We conducted a retrospective review of three cases of femoral neck fractures treated operatively in patients with osteogenesis imperfecta (OI). All of our patients had acetabular protrusio as well as coxa vara deformity. All patients successfully underwent cannulated screw fixation without avascular necrosis (AVN). One patient required a sub-adductor hip arthrogram during a revision procedure to assist with visualization of the proximal femur which resulted in improved screw purchase.

Conclusions: We report on three cases of femoral neck fractures in OI patients and a previously undescribed technique; using a hip arthrogram to assist in fluoroscopic evaluation of the proximal femur in these osteopenic patients.
\end{abstract}

Key Words: Osteogenesis imperfecta, Pediatric femoral neck fractures, Hip arthrogram

\section{INTRODUCTION}

Osteogenesis imperfecta (OI) is a genetic disorder of type I collagen synthesis. Frequently this is due to mutations in the COL1A1 and COL1A2 genes. ${ }^{[1]}$ Important orthopedic manifestations include increased bone fragility with frequent fractures. There is a broad phenotype with this disease ranging from perinatal lethality to severe deformities of the limbs and spine to near normal height and a mild predisposition to fractures. The Sillence criteria are frequently used to classify OI severity. ${ }^{[2]}$

Femoral neck fractures in the pediatric population are a rare entity, making up less than $1 \%$ of all pediatric fractures. ${ }^{[3]}$ These are frequently the result of either high energy trauma in healthy bone or minimal trauma in pathologic bone. ${ }^{[4]}$ The
Delbet system is used to classify these fractures in children. Frequent complications of these fractures include avascular necrosis, coxa vara, premature physeal closure, and leg length discrepancy. ${ }^{[5]}$

Treatment of femoral neck fractures in young patients with OI are rarely reported in the literature. There are additional factors that complicate surgical management in this patient population including poor bone quality, difficult radiographic visualization of the proximal femur secondary to deformity and severe osteoporosis, small patient size, and the frequency of preexisting implants. ${ }^{[6]}$

Within our institution, we report three cases of femoral neck fractures that occurred in patients with OI, and describe a technique of improving fluoroscopic visualization of proxi-

\footnotetext{
*Correspondence: Matthew D. Abbott; Email: mdabbott@med.umich.edu; Address: Department of Orthopaedic Surgery, Michigan Medicine, Ann Arbor, MI, Unites States.
} 
mal femoral anatomy in patients with severe osteopenia.

\section{Presentation}

\subsection{Case 1}

A 15-year-old male patient with OI Type III sustained multiple fractures following a motor vehicle collision. He sustained a right Delbet type II femoral neck fracture in addition to multiple fractures to bilateral femora and his right humerus (see Figure 1). He previously had Bailey-Dubow telescoping nails (Zimmer, Warsaw, IN/USA) placed in his bilateral femora. Operative management of his right femoral neck fracture was performed on the day of injury. A guide wire was percutaneously placed across the femoral neck into the femoral head, a single $4.5 \mathrm{~mm}$ cannulated screw was inserted, and adequate fixation was achieved (see Figure 2). The patient was made non-weight-bearing. Prior to this hospital admission the patient had been made "do not resuscitate" secondary to severe restrictive lung disease. He was eventually discharged to home hospice. No further clinical follow up was obtained and the patient died later the following year.

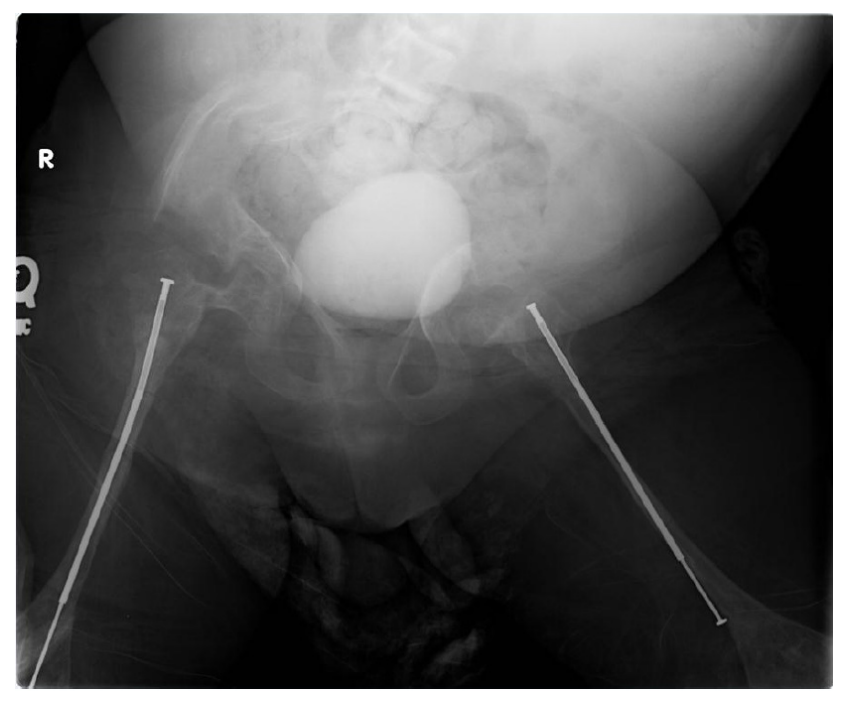

Figure 1. Case 1 - a right sided Delbet Type II femoral neck fracture was identified on radiographs

\subsection{Case 2}

A 26-year-old female with OI type III fell off of a step and landed onto her left knee resulting in groin pain and inability to bear weight. She was taken to the ED where she was found to have a left basicervical femoral neck fracture that was minimally displaced (see Figure 3 ). She was taken the same day to the operating room for percutaneous fixation. She previously had Rush rods (Zimmer, Warsaw,IN/USA) placed in her bilateral femora. Two guide pins were placed posterior to the Rush rod on the injured extremity and two $4.5 \mathrm{~mm}$ cannulated screws were used for fixation. A capsuPublished by Sciedu Press lotomy was also performed. She progressed well and at 3 months post-operatively was made weight bearing as tolerated. At three year follow up she had no evidence of avascular necrosis (AVN) or of worsening coxa vara deformity (see Figure 4).

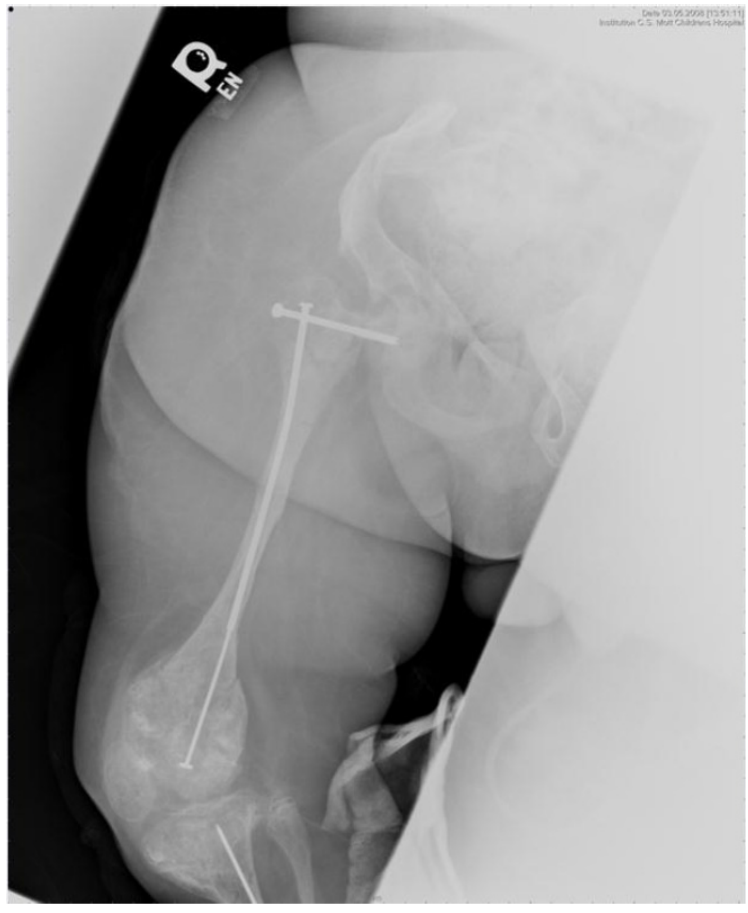

Figure 2. Case 1 - Fixation was achieved with a single $4.5 \mathrm{~mm}$ cannulated screw

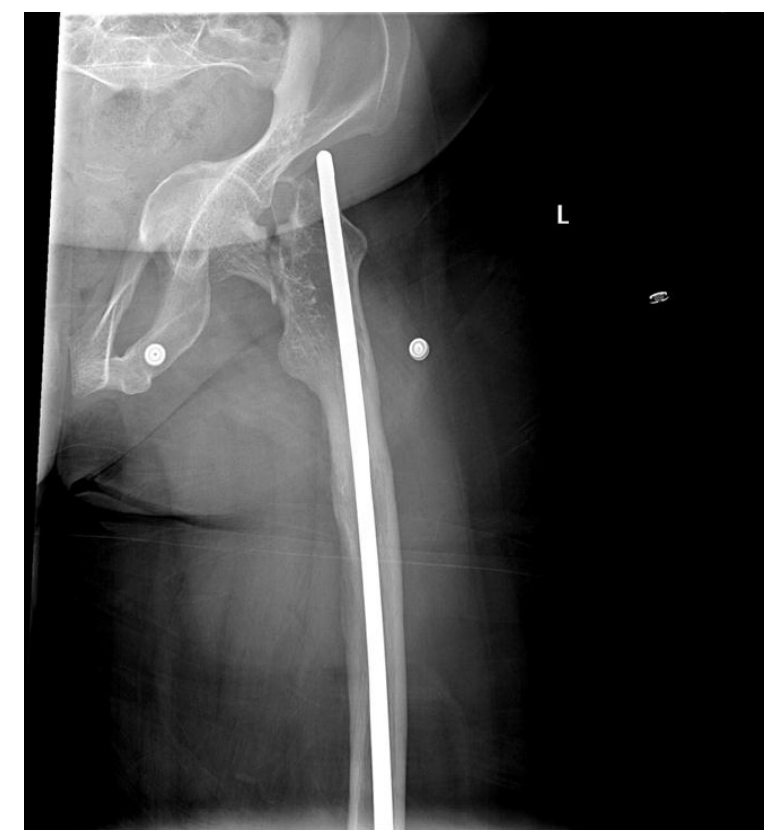

Figure 3. Case 2 - Radiographs reveal a left Delbet type III femoral neck fracture 


\subsection{Case 3}

A 5-year-old male presented from outside of the United States via medical visa and was diagnosed with OI type XI. He had Rush rods placed bilaterally to his femora for deformity correction with plans to return to his home country. While working with PT and walking for the first time in years, he was noted to have acute onset hip pain without any known trauma. Radiographs were obtained which revealed a right Delbet type III femoral neck fracture with minimal displacement (see Figure 5). Percutaneous fixation was performed with two $3.5 \mathrm{~mm}$ cannulated screws directed posterior to the intramedullary nails (see Figure 6). He was placed in a spica cast post-operatively. During routine-radiographs at his one-month check, it was noted that the screws had cut out anteriorly and superiorly likely due to poor screw placement (see Figure 7). Revision surgery was performed the following day. A percutaneous technique was utilized to remove the retained screws (see Figure 8). Similar to the index procedure, fluoroscopic visualization of the femoral head and neck was difficult due to the patient's osteopenia and proximal femoral deformity. Therefore, a sub-adductor hip arthrogram was performed using 50\% diluted contrast dye which helped significantly with visualization (see Figure 9). ${ }^{[7]}$ Two guide pins were then placed following adequate reduction of the fracture. Two $3.5 \mathrm{~mm}$ cannulated screws were then seated into this new trajectory (see Figure 8). The patient was placed into a hip spica cast post-operatively given the tenuous nature of the fixation. The spica cast was removed at one month post-operatively and at six weeks from the revision procedure he was made weight-bearing-as-tolerated. Radiographs at 5-month follow-up reveal no evidence of avascular necrosis and a coxa vara deformity that is unchanged from pre-injury imaging (see Figure 10).

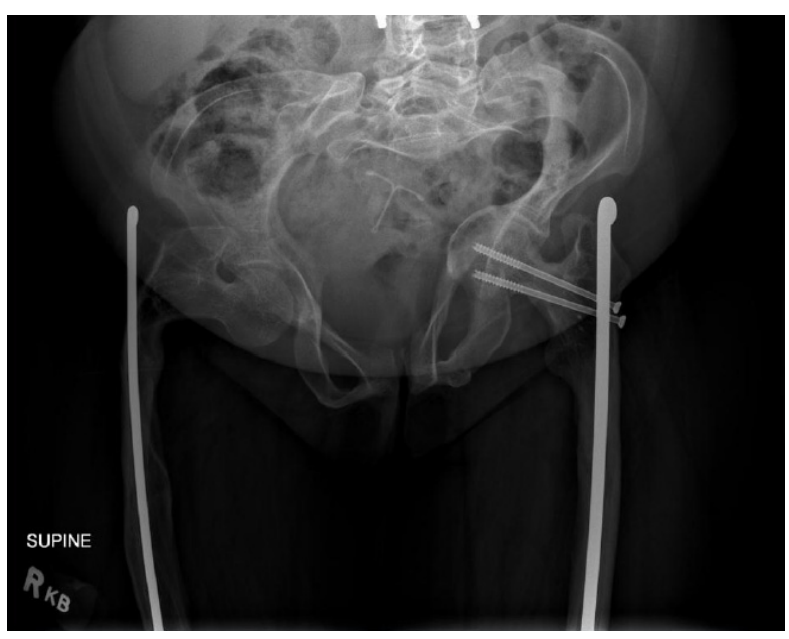

Figure 4. Case 2 - At 3 months post op there is no evidence of AVN or of worsening coxa vara

Two $4.5 \mathrm{~mm}$ cannulated screws were used for fixation

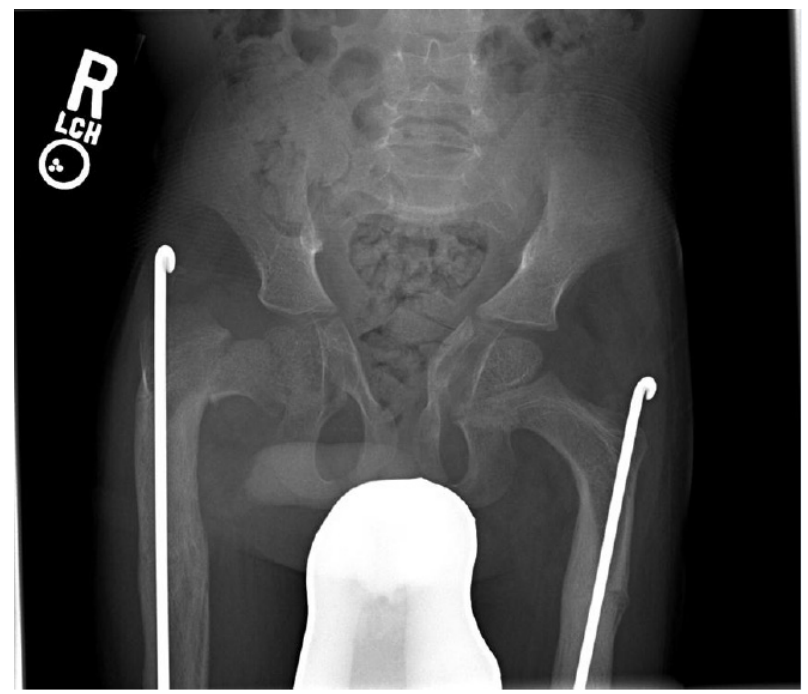

Figure 5. Case 3 - AP radiograph reveals a right sided Delbet type III femoral neck fracture with minimal displacement

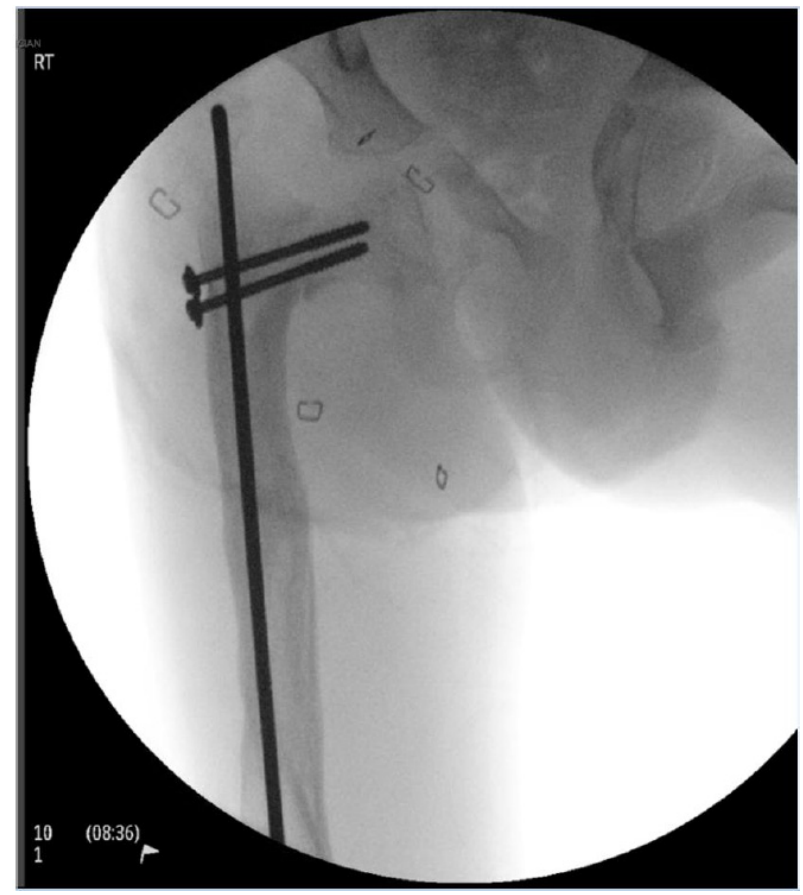

Figure 6. Case 3 - Intraoperative fluoroscopy was used to place two $3.5 \mathrm{~mm}$ cannulated screws posterior to the intramedullary nail

\section{Discussion}

Femoral neck fractures are rare injuries in a young population and patients with OI are no exception. In a review of records at our institution, only 3 cases of femoral neck fractures in OI patients have undergone operative fixation within the past 17 years. Historically the Sillence classification has been used to describe OI with type I being a mild form of the disease, 
type II being defined by extreme bone fragility and perinatal death, type III having severe bone fragility with extremity deformity and type IV of intermediate severity. ${ }^{[2]}$ Additional types ( V-XI) have been added to the classification system and account for a minority of the total OI cases. ${ }^{[8]}$ There is literature suggesting that specific types of OI patients more commonly have acetabular protrusio and an increased risk of proximal femur fractures. ${ }^{[9]}$ This was true of our patient population as all three of our patients had protrusio on AP pelvis radiographs. All three of our patients also had coxa vara deformity prior to their fractures although none developed a worsening of this post operatively which has been previously described in the literature. ${ }^{[10,11]}$ AVN is another feared complication in pathologic femoral neck fractures in young patients and in our series no patients developed this. ${ }^{[4,11]}$

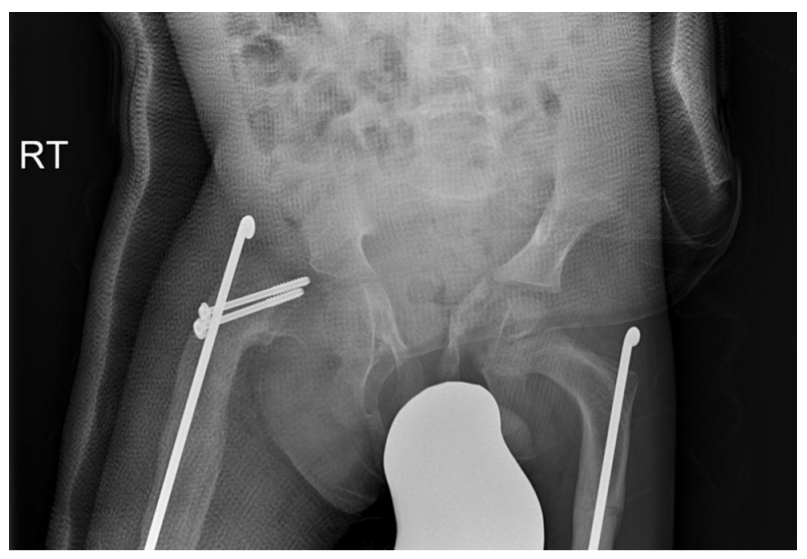

Figure 7. Case 3 - Routine post op imaging demonstrates screw cut out anterior and superior with loss of fixation

OI patients with femoral neck fractures often have poor bone quality and small areas for implant purchase with pre-existing hardware and dysmorphic proximal femurs, making it difficult to achieve adequate fixation. In case 3 , revision surgery had to be performed likely due to poor screw placement during the index procedure as a result of difficult radiographic visualization. Cannulated screw fixation has been used successfully in other case series without complications typically associated with femoral neck fractures in young patients. ${ }^{[10-13]}$ Our patients all underwent cannulated screw fixation without AVN, or worsening coxa vara. Although our limited follow up time is a weakness of this series. In case 3 we were able to revise our cannulated screws into an area of better purchase after utilizing an intraoperative hip arthrogram to improve our visualization of the femoral head and neck anatomy. This is a previously undescribed technique in conjunction with femoral neck fracture procedures and could be useful in any patient with severe osteopenia.

Published by Sciedu Press

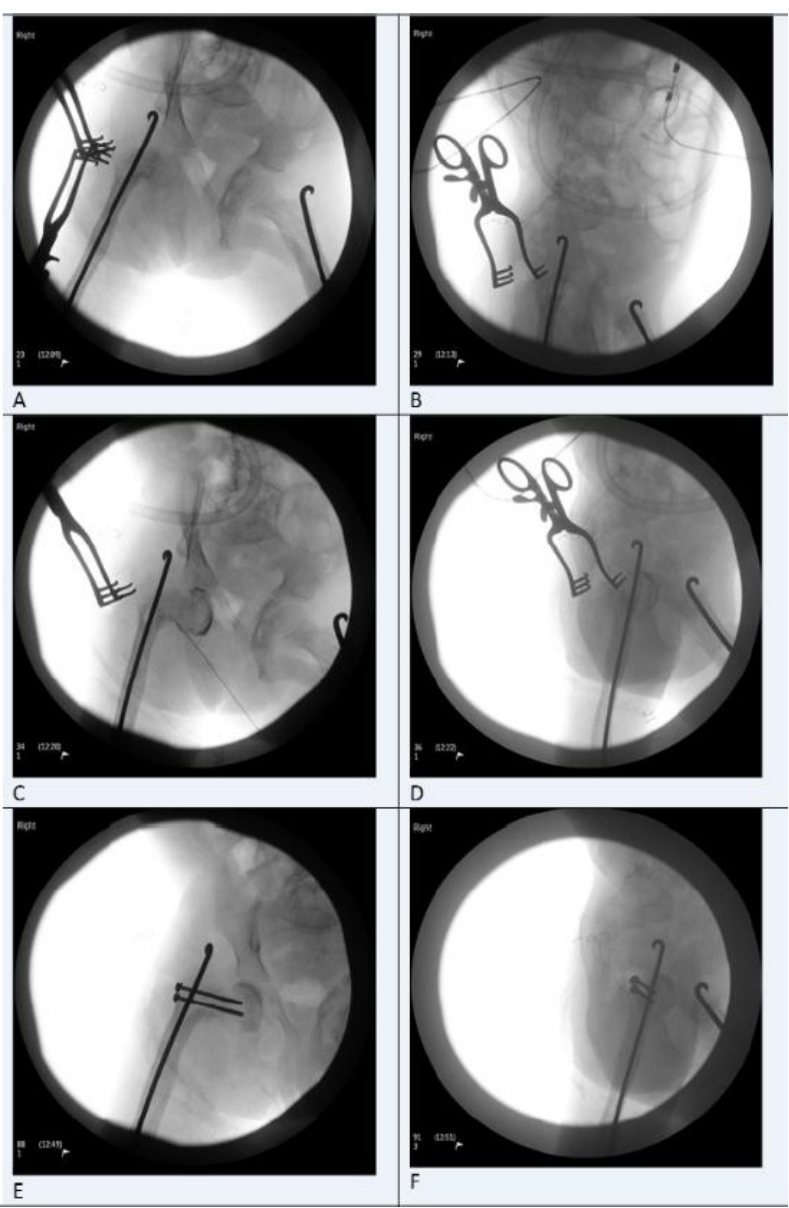

Figure 8. During the revision procedure, visualization of the proximal femoral anatomy was difficult secondary to the patient's severe osteopenia and deformity (A, B). A sub-adductor hip arthrogram was performed with improvement in anatomic visualization $(\mathrm{C}, \mathrm{D})$. The screws were placed with improved bone purchase and the patient was placed into a hip spica cast $(\mathrm{E}, \mathrm{F})$.

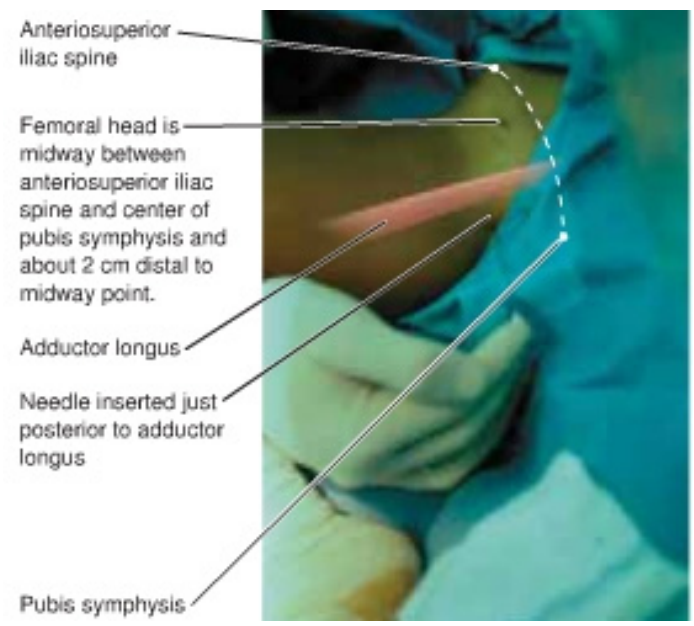

Figure 9. Visual representation of a sub-adductor arthrogram with anatomic landmarks labeled ${ }^{[7]}$ 


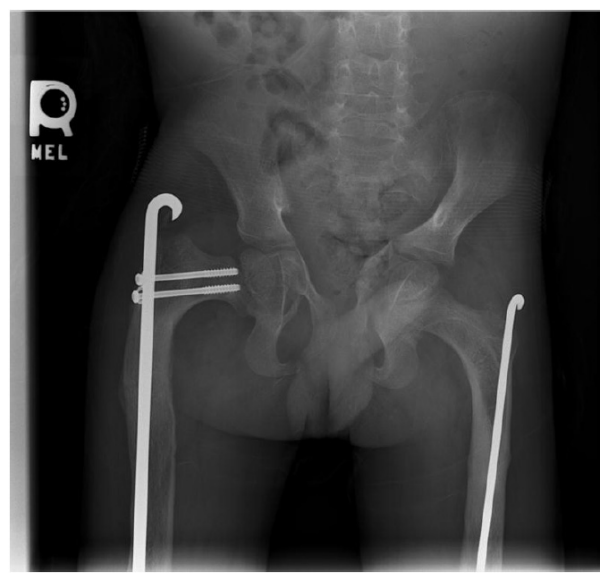

Figure 10. Case 3 - At 5 month follow-up there is no evidence of AVN or worsening coxa vara (the fixation construct remained stable)
Although rare, femoral neck fractures in OI patients can be treated similarly to femoral neck fractures in other populations; with reduction and internal fixation. This can be successfully done using cannulated screw fixation with few complications. However, due to anatomic abnormalities, osteopenia, and pre-existing implants, use of intra operative fluoroscopy can often be very challenging with poor visualization. The introduction of contrast dye into the hip capsule at the time of fixation can greatly improve visualization. This technique may be helpful for other patient populations with osteopenia and proximal femoral deformity.

\section{Conflicts of InTERest Disclosure}

The authors declare they have no conflict of interest.

\section{REFERENCES}

[1] Rauch F, Glorieux FH. Osteogenesis imperfecta. Lancet. 2004; 363: 1377-1385. https://doi.org/10.1016/S0140-6736(04 ) 16051-0

[2] Sillence DO, Senn A, Danks DM. Genetic heterogeneity in osteogenesis imperfecta. Journal of Medical Genetics. 1979; 16: 101-116. PMid: 458828. https://doi.org/10.1136/jmg.16.2.101

[3] Cheng JC, Tang N. Decompression and stable internal fixation of femoral neck fractures in children can affect the outcome. Journal of Pediatric Orthopedics. 1999; 19: 338-343. https://doi.org/10 .1097/01241398-199905000-00010

[4] Nayeemuddin M, Higgins GA, Bache E, et al. Complication rate after operative treatment of paediatric femoral neck fractures. Journal of Pediatric Orthopaedics B. 2009; 18: 314-319. PMid: 19730403. https://doi.org/10.1097/BPB.0b013e32832d5d5f

[5] Azouz EM, Karamitsos C, Reed MH, et al. Types and complications of femoral neck fractures in children. Pediatric Radiology. 1993; 23 : 415-420. PMid: 8255640. https://doi.org/10.1007/BF0201 2436

[6] Chow W, Negandhi R, Kuong E, et al. Management pitfalls of fractured neck of femur in osteogenesis imperfecta. Journal of Children's Orthopedics. 2013; 7: 195-203. PMid: 24432078. https : //doi.org/10.1007/s11832-013-0495-6
[7] Wiesel SW. Operative techniques in orthopaedic surgery. Wolters Kluwer Health/Lippincott Williams \& Wilkins; 2011. 1510 p.

[8] Forlino A, Cabral WA, Marini JC. New Perspectives on osteogenesis imperfecta. Nature Reviews. Endocrinology. 2011; 7: 540-557. PMid: 21670757. https://doi.org/10.1038/nrendo.2011.81

[9] Trehan SK, Moraki E, Raggio CL, et al. Acetabular Protrusio and Proximal Femur fractures in patients with osteogenesis imperfecta. Journal of Pediatric Orthopedics. 2014.

[10] Chung FC, Chong KW, Koon WM. A case of screw fixation of femur neck fracture with the use of computer navigation in a woman with osteogenesis imperfecta. The Journal of Trauma. 2008; 65: 1168 1170. PMid: 18277290. https://doi.org/10.1097/01.ta.00 $00197149.09525 .9 \mathrm{~b}$

[11] Livesley PJ, McAllister JC, Catterrall A. The treatment of progressive coxa vara in children with bone softening disorders. International Orthopaedics. 1994; 18: 310-312. PMid: 7852013. https://doi.org/10.1007/BF00180233

[12] Tsang KS, Adedap A. Cannulated screw fixation of fracture neck of femur in children with osteogenesis imperfecta. Journal of Pediatric Orthopedics B. 2011; 20: 287-290. PMid: 21606853. https: //doi.org/10.1097/BPB.0b013e328347a345

[13] Shrader MW, Schwab JH, Shaughnessy WJ. Pathologic femoral neck fractures in children. American Journal of Orthopedics. 2009; 38: 83-86. PMid: 19340370. 